\title{
géotechnique et environnement : leur prise en compte pour l'aménagement de l'agglomération nantaise
}

\author{
allocution d'accueil \\ de la municipalité de Nantes \\ et de ses Services Techniques \\ aux deuxièmes journées nationales géotechniques
}

\author{
par \\ A. Mahé \\ Maire-Adjoint, Ville de Nantes \\ P. Fontalirand \\ Services Techniques, Ville de Nantes
}

\begin{abstract}
« Depuis des millénaires, la Ville tient une place prépondérante dans l'histoire des civilisations : Babylone, Athènes, Rome, Paris, Moscou, Pékin, Londres, New-York, Brasilia sont autant de jalons du phénomène urbain.
\end{abstract}

La Ville est donc, depuis l'origine des temps, considérée comme la projection de la société tout entière.

Ce phénomène urbain résulte d'une fascination certaine des hommes par la Ville et se caractérise par une concentration de population dans des cités, qui offrent à la fois : travail, logement, loisirs et libertés de choisir un mode d'expression, de rencontres et d'échanges.

Mais la connaissance du passé d'une ville, l'examen de ses évolutions successives, du comportement de ses habitants, au cours de périodes troublées ou de brusques changements, permet souvent de bien comprendre le présent et, donc, de mieux appréhender l'avenir.

Car il est bien certain qu'à côté de tous les témoignages du passé, marqués dans la pierre, le « contenu social " intéresse, au plus haut point, les édiles chargés de "gouverner " la Ville.

$$
* * *
$$

Pour sa part, NANTES bénéficie d'un passé prestigieux.

Son origine remonte à plus de 1000 ans avant J.C.; des navigateurs s'installent au confluent de la Loire et de l'Erdre, alors soumise à l'influence des marées.
Nul doute que le site a joué un rôle primordial dans la recherche de l'emplacement de sa fondation : c'est en effet, ici, que le franchissement de la Loire, entre la mer et l'amont, est le plus aisé, grâce aux innombrables petites îles qui jalonnent la traversée du fleuve du nord au sud.

A cette convergence d'éléments hydographiques : Loire, Erdre, Sèvre, se surimpose ensuite celle de voies terrestres, la plupart d'origine gallo-romaines, déterminant ainsi une fonction commerciale et d'échanges jamais démentie depuis.

Pour assurer sa réussite, la Ville se dote de plusieurs enceintes successives :

- la première, gallo-romaine, et d'une longueur de $1600 \mathrm{~m}$ environ, couvre une superficie de $16 \mathrm{ha}$, - la deuxième est élevée dans le faubourg du Marchix, en 1592, sous l'impulsion du Duc de Mercœur.

Et les épisodes ne manquent pas, qui font alterner heures sombres et de grandeur.

NANTES doit aux Ducs de Bretagne, qui en firent leur capitale, six siècles de fastes et d'indépendance.

Mieux, Anne, Duchesse de Bretagne, fut, par ses mariages successifs avec Charles VIII et Louis XII, deux fois Reine de France.

Notre château, qui est le résultat de l'apport architectural successif de quatre siècles, du XIIIème au XVIème, allie la rigueur de l'âge féodal à l'imagination raffinée de la Renaissance. 
La cathédrale de NANTES est un des autres joyaux historiques de la Ville ; la première pierre est posée en 1434 ; la nouvelle façade gothique, placée devant la cathédrale romane est due à l'architecte Mathelin Rodier.

La fin du XVIIème siècle et le XVIIIème siècle représentent l'âge d'or de la Ville fondé sur ce qu'on appelle le trafic du "bois d'ébène ", autrement dit des esclaves. NANTES est le premier port français. C'est de cette période de richesse qu'est née, sous l'impulsion de l'architecte Ceineray, puis de son disciple Crucy, la reconstruction de tous les quais du fleuve et de leurs bâtiments, ainsi que de la construction, à partir de 1721, sur l'tle Feydeau, de maisons d'armateurs à l'architecture opulente.

$\mathrm{Au}$ XIXème siècle, la Ville se tourne résolument vers l'industrie, notamment métallurgique et alimentaire (biscuiterie) ; l'alimentation en eau potable est assurée et le réseau public d'assainissement commence à fonctionner.

$\mathrm{Au}$ cours de la première moitié du siècle actuel, deux grands travaux édilitaires sont menés à leur terme :

- la traversée de la Ville dans le sens Est-Ouest, en souterrain et en tranchée, par les lignes S.N.C.F.,

- le remblaiement de deux bras de Loire et de celui de l'Erdre, dont le cours est dévié par un canal souterrain du quai Ceineray au quai Malakoff.

La Ville sort meurtrie de la deuxième guerre mondiale ; les bombardements aériens de 1943 ont paralysé le port et défiguré la cité ; 1525 hectares bombardés sur 6500,7000 logements détruits partiellement ou en totalité, plusieurs milliers de victimes.

Après la période de reconstruction, apparaît l'âge d'or de la construction. A partir d'un développement économique rapide et continu et d'un accroissement démographique sans précédent, la périphérie se couvre de lotissements, puis d'immeubles collectifs et, enfin, de grands ensembles qui modifient sensiblement la silhouette urbaine de la Ville, hormis le centre.
La crise du logement étant très aiguë, la prépondérance est donnée au "quantitatif ", au détriment des aspirations sociales des habitants.

Les activités, enserrées en tissu urbain, éclatent littéralement ; des zones industrielles nouvelles sont créées dans l'agglomération et les communes limitrophes.

L'université, au Nord de NANTES, se développe aussi rapidement.

Mais, à partir des années 1975-1980, l'aspect qualitatif est enfin pris en compte.

D'autre part, la crise du pétrole, la longueur et le coût des trajets quotidiens domicile-travail, parfois l'insuffisance des équipements publics périphériques, la difficulté de créer une vie sociale et, d'autre part, le refus nettement marqué de la population vis-à-vis des "grands ensembles " impersonnels, aux espaces mornes et indifférenciés, ont conduit tout naturellement une partie de la population à rechercher, sinon le centre-ville, du moins son pourtour immédiat.

Aux grandes opérations de construction doivent donc se substituer, maintenant, plusieurs types d'actions nouvelles :

- la construction de lotissements ou de petits immeubles collectifs, plus respectueux que par le passé de l'environnement immédiat et bien intégrés dans les quartiers; notamment, la construction de logements sociaux, afin d'assurer l'indispensable diversité sociale au centre-ville, doit être prévue ; des résultats ont déjà été obtenus,

- la réhabilitation de logements (notamment dans le centre-ville) dans des immeubles dont le gros-œuvre est souvent sain ; il s'agit de mieux les adapter aux impératifs de l'habitat moderne et de les doter des éléments de confort (isolation thermique, acoustique, salle d'eau, chauffage...),

- la réhabilitation des logements H.L.M. d'aprèsguerre construits très rapidement et à un 'moindre coût ; l'action devra porter tant sur le bâti que sur les espaces et équipements environnants.

Pour y parvenir, c'est une véritable politique urbaine qui doit être mise en place, intégrant 
également d'autres actions sur les transports collectifs urbains, les circulations tous modes, le stationnement, la vie associative, sociale et culturelle, la promotion du centre-ville.

Les élus nantais sont conscients des difficultés actuelles, mais ils entendent agir, à partir d'une connaissance approfondie du développement urbain, avec courage, persévérance et ténacité pour que cette politique se traduise dans les faits ; ils ne veulent pas d'opérations prestigieuses et coûteuses, mais tout un enchaînement d'actions plus modestes, concourant à la même finalité et répondant aux diverses aspirations sociales de la population.

Enfin, toute une réflexion est conduite depuis plus d'un an sur, non pas la seule Ville de NANTES, mais à l'échelle de l'agglomération de fait, c'est-àdire sur une quinzaine de communes. "

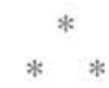

Sans doute, Messieurs, allez-vous trouver que je suis loin du sujet de vos préoccupations.

Mais j'ai tout d'abord tenu à vous brosser un très rapide tableau de la Ville que vous avez fort aimablement choisie pour le déroulement de ces Deuxièmes Journées Nationales Géotechniques.

J'en viens maintenant à votre sujet, en vous présentant les dessous de notre Cité.

Citant Mme Ters et MM. Marchand et Weecksteen, co-rédacteurs de la notice introductive de la carte géologique de Nantes, dressée par la B.R.G.M. sur les documents de l'I.G.N., je dirai, avec eux, que celle-ci fait apparaître, dans sa partie N.W., le plateau granitique du Sillon de Bretagne, s'abaissant d'Ouest en Est, du Temple vers Orvault et de Saint-Etienne-de-Montluc à Chantenay (de 91 à $70 \mathrm{~m}$ ).

De part et d'autre du couloir ligérien, s'étendent de basses plates-formes, entre 36 et $20 \mathrm{~m}$, dominées par le talus tectonique du Sillon de Bretagne, au N.E., et par celui de Saint-Père-en-Retz, au S.W. (feuille Nantes - Ile du Pilier au 1/80 000).
A l'Est, une région affaissée, occupée par des micashistes, s'étend, vers $30 \mathrm{~m}$ d'altitude, de part et d'autre de la vallée de l'Erdre, qui fut entièrement occupée par la mer pliocène. Dans l'angle N.E. de la feuille (les Cinq-Chemins), cette zone déprimée se trouve dominée par le Plateau de 70 - $80 \mathrm{~m}$ qui fait suite au Sillon de Bretagne et s'étend au Nord de Mauves (feuille Vallet).

Le couloir de la Loire, colmaté par les alluvions récentes et anciennes, est relativement étroit à la traversée de formations telles que les roches granitiques du Sillon de Bretagne, les gneiss leptynitiques et les roches basiques, entre la Martinière et Bouguenais. Il s'élargit, à l'Ouest de la feuillle, en direction de la Grande-Brière, et à l'Est, vers la zone affaissée de Mauves - La Chapelle-Basse-Mer.

Le socle ancien est représenté, sur cette feuille, par des granites et les formations cristallophyliennes fortement plissées du flanc N.E. de l'anticlinorium de Saint-Nazaire - Mervent (micashistes, gneiss leptyniques et roches basiques : amphibolites et serpentinites).

Le style de plissement qui les affecte est souvent isoclinal, à pendage N.E. dominant, et les couches sont fortement redressées : on observe aussi des ondulations molles, à pendages faibles (entre Saint-Jean-de-Boiseau et Brains).

A l'Est de la feuille, les micashistes albitiques dessinent une structure péri-anticlinale, à contour elliptique, d'orientation E.W., avec plongement d'axe vers l'Est.

Un important accident coupe obliquement la vallée de la Loire, au N.W. et au S.E. de Saint-Sébastiien, dans la zone d'affrontement entre les micasiistes albitiques de Saint-Sébastien et la bordure ouest du pli couché de Champtoceaux.

Les restes des dépôts de la transgression pliocène sont abondamment représentés sur cette feuille, sous la forme de nappes de sables et de cailloutis. Ces terrains ont fossilisé un relief qui était très voisin de l'actuel. Les limons quaternaires et les alluvions anciennes et modernes de la Loire recouvrent une partie importante des autres formations. 
Contrairement à ce que l'on pourrait croire, l'évolution de notre Ville n'a pas été influencée par la nature de son sous-sol, mais a été conditionnée par les éléments naturels qu'elle comporte, en particulier, par ses fleuves et rivières, comme le montrent les schémas ci-joints.
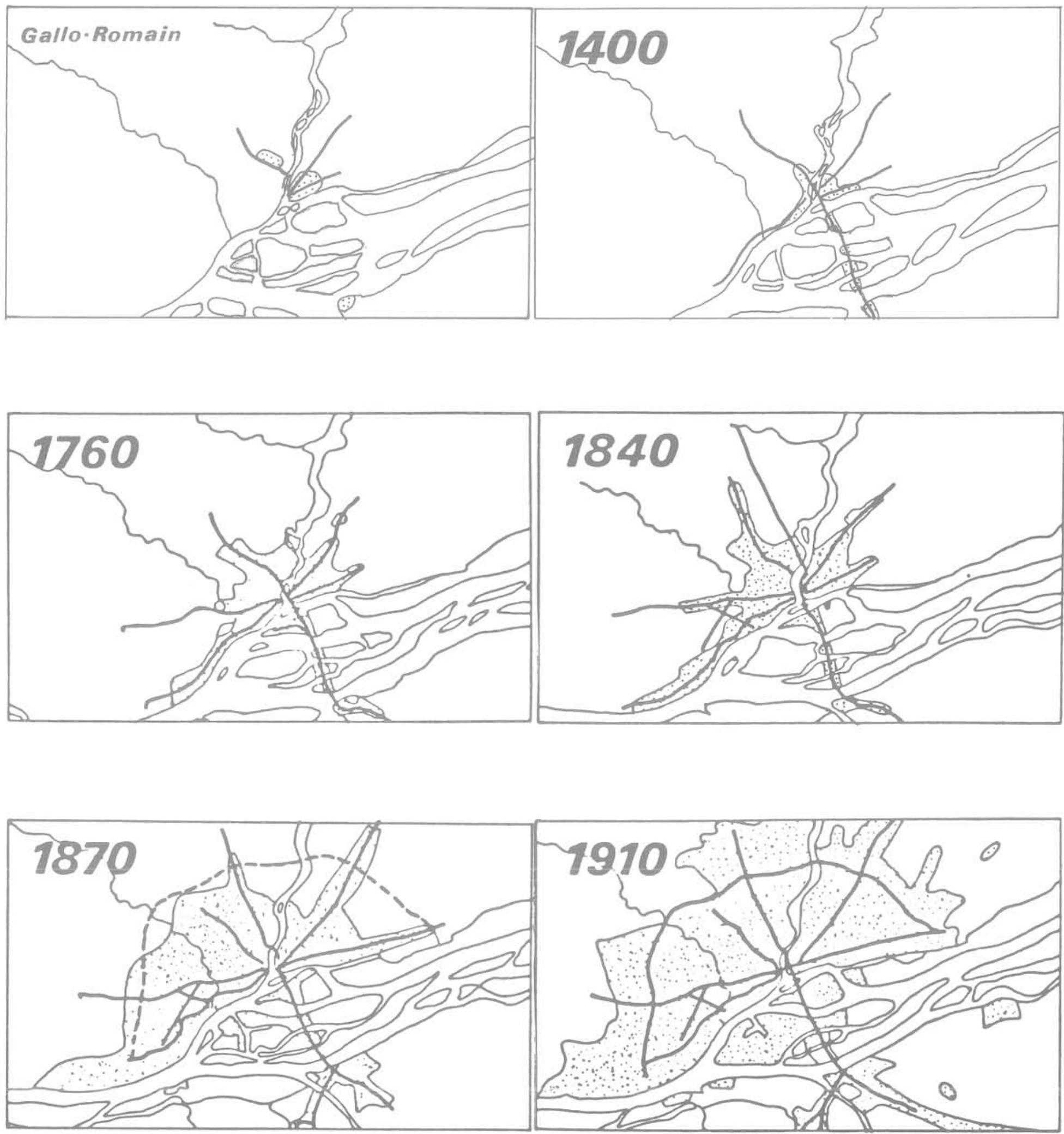

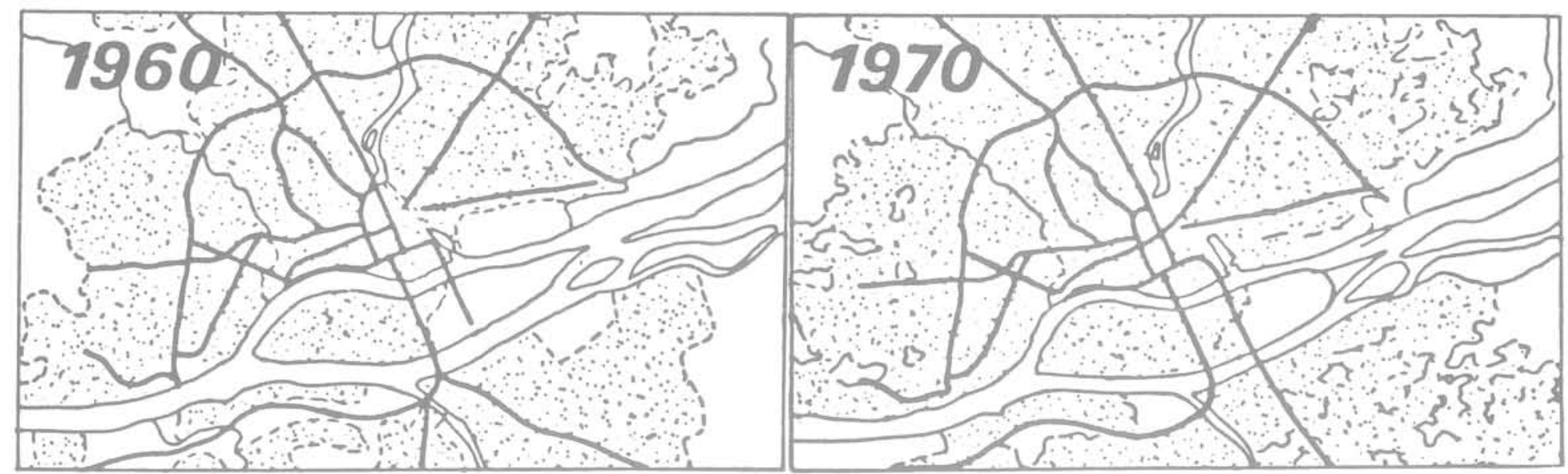

Il est donc flagrant que notre Ville s'est développée de façon à la fois concentrique autour du noyau initial et de façon linéaire le long de ses voies d'eau et de ses routes, quelle que soit la nature de son sous-sol ; les deux exemples qui peuvent illustrer mon propos sont l'Ile Feydeau et le quai de la Fosse, où ont été édifiés, sur pieux en bois, selon un procédé inventé par l'architecte Pierre Rousseau, quelques-uns des plus luxueux immeubles de Nantes.

Héritiers du passé, nous nous trouvons, encore actuellement, et je dirai même, de plus en plus, confrontés au problème du développement de notre Ville sur des sites qui, pour nombre d'entre eux, se présentent comme des cas particuliers appelant des solutions particulières.

Je demanderai à M. Fontalirand, Directeur Général des Services Techniques de la Ville de Nantes, de bien vouloir en expliciter quelquesunes.

$$
\text { * * }
$$

Le thème no 1 de vos Journées Géotechniques s'intitule : "Prise en compte de l'environnement dans les études, la conception et l'exécution des ouvrages et travaux $»$.

J'ai commencé par m'interroger : géotechnique et environnement? Deux termes qui semblent, a priori, bien éloignés l'un de l'autre à l'instant où se présente, pour les spécialistes que nous sommes des techniques urbaines, un problème à résoudre. Puis, à la réflexion, nous nous apercevons que, comme M. Jourdain faisait de la prose sans le savoir, la géotechnique s'est imposée à nous, presqu'à notre insu, par l'influence directe ou indirecte qu'elle peut avoir sur l'environnement et réciproquement.

Je vais, en conséquence, me permettre de passer très rapidement en revue quelques exemples de ce que nous avons pu rencontrer à Nantes.

Je ne citerai que pour mémoire, deux importantes réalisations : la rocade dans sa partie N.E. et la station de traitement des eaux usées de la partie Nord de l'agglomération, ces deux sujets devant être traités par MM. Gonin et Pfeff́er, de la Société SIMECSOL ; il est évidemment inutile de vous infliger deux fois le même pensum.

\section{Mon propos comportera trois parties :}

- aménagement de zones inondables - modification du site,

- aménagement d'une carrière en zone de loisirs,

- construction d'ouvrages d'art.

\section{Aménagement de zones inondables - Modifi- cation du site}

M. le Maire-Adjoint Mahé vous a rappelé que Nantes est drainée par de nombreux fleuves, rivières et cours d'eau ; de ce fait, nous possédions, et possédons d'ailleurs encore, des zones inon- 
dables, généralement marécageuses qui, outre qu'elles dégradaient le site, tant par leur aspect que par leurs nuisances, occupaient des surfaces importantes inutilisables. La première nécessité qui s'est imposée fut de les remblayer, sans que pour autant, dans la majorité des cas, leur destination future soit nettement définie. Il est d'ailleurs intéressant de noter que nous n'étions pas les premiers à modifier ainsi le site. Celui de la Loire le fut perpétuellement, de l'époque celtique à nos jours. L'obligation devant laquelle se trouvent toutes les Communes d'éliminer, au moindre coût, les résidus urbains d'origine ménagère ou industrielle, a conduit à la solution de facilité d'utiliser ceux-ci comme remblais, parfois complétés par des remblais hydrauliques en sable. Ces terrains, ainsi récupérés, ont eu deux destinations principales :

- des zones de construction,

- des zones de loisirs.

\section{Zones de construction}

Dans ce cas, se pose le problème crucial du choix des fondations. Les terrains remblayés étant, naturellement, de très mauvaise portance, les remblais apportés ne leur retirent ni ne leur apportent rien, d'où la nécessité, tant pour des motifs techniques qu'économiques, soit de fonder à de grandes profondeurs pour des surcharges importantes, soit de rechercher un allègement des constructions permettant des fondations superficielles. Il en résulte un choix ayant une conséquence indéniable sur un paysage urbain futur. Deux Z.U.P. ont ainsi été réalisées : Beaulieu et Pirmil. Dans les deux cas, pour des motifs susexprimés, le programme a comporté essentiellement la construction d'immeubles hauts, parfois de tours, sur fondations profondes. Afin de rompre avec cet horizon de béton, rarement agréable à l'œil par suite d'un manque de recherche architecturale, l'achèvement de la Z.U.P. de Beaulieu, actuellement en cours, se réalise selon un plan d'épannelage permettant de construire des immeubles bas sur fondations superficielles. Ce nouveau paysage rejoint, d'ailleurs, les nouvelles aspirations de la population. Nous voyons donc bien là l'influence de la géotechnique sur l'urbanisation.

\section{Zones de loisirs}

L'ampleur des problèmes posés par les fondations dans les zones récupérées par remblais nous a conduits à affecter la majorité de nos autres zones de remblais à des équipements de sports et de loisirs.

- Tout d'abord, le terrain de camping du Petit-Port, réalisé sur des remblais de terre avec ses bâtiments légers : accueil - sanitaires.

Compte tenu de la nature du remblai et malgré son épaisseur de 2 à 4 mètres, nous n'avons pas eu pratiquement de tassement de sol, ni de trouble dans les ouvrages de superstructure ou d'infrastructure : par ailleurs, nous n'avons rencontré aucun échec végétal.

- Ensuite, l'ensemble sportif de Beaulieu : nous nous trouvions devant un remblai mixte, par zones : résidus urbains de toutes natures et sable.

Compte tenu de l'ancienneté du remblai (1947 à 1964), nous n'avons constaté aucun mouvement. Par contre, au moment du semis du gazon, il est apparu que des zones ont rapidement disparu ; les zones brûlées se sont manifestées sur la seule partie remblayée en résidus urbains et, de plus, au droit des canalisations de drainage et des fentes de suintement remplies d'agrégats fins ; les émanations gazeuses du sous-sol, dont la fermentation a été ranimée par les diverses interventions faites dans le remblai : dégagement de gaz toxiques (méthane) et entraînant une élévation de la température, ont été la cause de ces dégradations qui ont progressivement disparu.

- Place Esnoul-des-Châtelets : nous traiterons cette réalisation sous la rubrique " aire de loisirs ", bien qu'il s'agisse de la création d'un marché de plein air sur remblais d'ordures ménagères.

Si nous n'avons enregistré que peu de mouvements de sol, nous avons, par contre, constaté des effets catastrophiques sur les plantations : les travaux effectués en sous-sol et dans un remblai récent ont " réveillé " la fermentation entraînant pendant cinq années le remplacement d'arbres morts, parfois jusqu'à $60 \%$ l'an.

- La plaine de jeux de Sèvres a été réalisée sur décharge d'ordures ménagères, à l'exclusion de tous déchets industriels.

Nous avons, là aussi, constaté des mouvements de sol et craignons de nous trouver confrontés aux conséquences susexprimés sur la végétation, au moment où seront réalisées les plantations. 
J'en terminerai avec ce type d'aménagement en citant une réalisation mixte exécutée sur des remblais de résidus urbains de 1960 à 1968, dans la zone de Malakoff.

Il s'agit de la construction :

- d'une piscine,

- d'un groupe scolaire,

- et d'un parc paysager.

Dans ce cas, un apport supplémentaire de remblais en matériaux inertes correctement compactés, d'une hauteur de 3 à 4 mètres au-dessus d'une ancienne décharge de résidus urbains, a permis de n'enregistrer ni tassement du sol, ni réaction sur les végétaux, les divers travaux que nous avons exécutés en infrastructure n'ayant pas atteint la couche des ordures.

On peut donc conclure que la récupération de zones inondables et marécageuses par remblaiement et leur affectation à des zones de loisirs est une bonne solution qui apporte une amélioration de l'environnement. Toutefois, la nature des remblais utilisés peut entraîner des déboires momentanés, à partir du moment où ceux-ci sont constitués par des ordures ménagères, et que au cours des travaux il soit nécessaire d'y effectuer des terrassements.

\section{2 - Aménagement d'une carrière en zone de loisirs}

Dans le cadre de la prise en compte de l'environnement dans la conception et de la réalisation des travaux, je n'aurai garde d'oublier qu'aux portes de Nantes, sur la commune de Moisdon-la-Rivière, existait une ancienne carrière présentant des excavations, buttes, rampes, socles de concasseurs et de trémies, le tout envahi plus ou moins par une végétation sauvage. Non seulement cette ancienne carrière dénaturait le site, mais elle présentait un danger pour qui s'y aventurait.

Après avoir exécuté des reprofilages et des comblements de fosses, tout en maintenant quelques reliefs, et en les intégrant, et en conservant la végétation spontanée intéressante, nous avons repris le front de taille et aménagé une aire de loisirs rustiques.

\section{3 - Projets d'ouvrages d'art}

Les solutions techniques à adopter sont fondamentalement influencées par la nature du sous-sol, et l'environnement doit, impérativement, être respecté au maximum, ceci indépendamment d'autres contraintes. Mais ces impératifs ne sont pas obligatoirement contradictoires et l'un peut venir au secours de l'autre.

Je citerai deux exemples :

\section{- Le pont des Bourdonnières sur la Sèvre}

Recherchant l'économie, notre première esquisse comportait un ouvrage d'une portée égale à la largeur de la rivière à franchir, soit 81 mètres avec deux piles en rivière et raccordement au terrain naturel par des remblais d'une hauteur allant de zéro à près de neuf mètres respectivement de $294 \mathrm{ml}$. et de $665 \mathrm{ml}$. de longueur de part et d'autre de l'ouvrage de franchissement.

Du rapport de sondage dont nous avons eu alors connaissance, nous pouvons extraire :

- du point de vue géologique, le socle rocheux est constitué de roches cristallophylliennes et métamorphiques au pendage fortement redressé $\left(60^{\circ}\right.$ par rapport à l'horizontale). Par la suite, au cours de la transgression du Flandrien, les "gouttières " de la Loire, de la Sèvre et de leurs affluents ont été remblayées par des dépôts fluviatiles ou fluviomarins, le cours d'eau se déplaçant d'un bord à l'autre de son lit majeur ; le tracé actuel de l'écoulement en période d'étiage ne se fait donc plus à la verticale du lit du torrent originel (paléolit).

Les terrains rencontrés au cours des sondages sont constitués par :

- des remblais,

- des alluvions, dans lesquelles on peut distinguer :

- des argiles limoneuses et limons sableux,

- des vases argileuses,

- des sables argileux,

- des produits d'altération du micashiste formant le substratum : limons sableux,

- le substratum rocheux.

La topographie du substratum, reconnue de manière globale par l'étude de sismique, est confirmés par les sondages. Le paléolit de la Sèvre ne se trouve pas exactement à l'aplomb de son cours actuel, mais un peu plus au Nord ; le substratum rocheux est rencontré à $26 \mathrm{~m}$ de profondeur. 
Celui-ci constitue une couche portante de très bonne qualité.

Les alluvions constituent les terrains de remplissage de la vallée de la Sèvre et de son petit affluent, le ruisseau de la Chaussée. On y distingue plusieurs faciès, en allant des terrains les plus anciens aux plus récents :

- des sables argileux :

La masse la plus importante tapisse le fond du paléolit de la Sèvre ; elle a une épaisseur de 9,50 m au maximum,

- des vases argileuses et tourbeuses :

Ces vases sont rencontrées, en règle générale, entre les cotes NGF + 2 et - 13 ; elles surmontent directement le rocher, lorsque celui-ci se situe au-dessus de la cote - 13. Ce sont des argiles plastiques limoneuses et sableuses, molles, micacées et de couleur gris-bleuté ; elles deviennent organiques en profondeur,

- de l'argile limoneuse et des limons sableux:

La partie supérieure des alluvions, sur une épaisseur de 1 à $2,50 \mathrm{~m}$, est constituée par un terrain un peu différent des vases sous-jacentes, soit une argile limoneuse marron clair à grise, avec des traces rouilles, micacée, assez compacte, soit un limon gris à marron clair, avec cailloutis siliceux, vasard et saturé, qui fait la transition avec les vases.

Nous nous trouvons donc en présence d'un sol non stratifié horizontalement.

Les calculs effectués nous montraient que, si nous utilisions la technique des remblais, l'évolution secondaire, après le tassement de la consolidation primaire, donnerait un supplément de tassement de $0,33 \mathrm{~m}$ au bout de dix ans, et nous conduirait à la nécessité d'une hauteur totale de remblais de $11 \mathrm{~m}$.
Les études de stabilité à court terme nous confirmaient la nécessité d'effectuer les remblais par couches successives ; pour ce qui concerne celles à long terme, la conclusion en était que la sécurité de la confection des remblais en bordure de la Sèvre ne pourrait être assurée de manière satisfaisante.

La nécessité de laisser à la Sèvre un débouché suffisant, en temps de crue, et de dégager un certain tirant d'air nous interdisait de détourner cette rivière pendant les travaux de remblaiement.

Nous nous trouvions, en outre, dans notre esquisse de base, devant la nécessité de construire trois ouvrages : franchissement de la Sèvre et des deux chemins encadrant celle-ci, le chemin Bleu et le chemin des Roches-Vertes. Il devenait aventureux d'envisager un projet comportant des remblais entre ces trois ouvrages, remblais dont la stabilité ne pouvait être assurée de façon satisfaisante et qui engendraient des efforts parasites sur les fondations des ouvrages d'art, par l'intermédiaire des terrains mous de leur assiette.

Ces constatations nous ont alors conduits à adopter une solution viaduc sur fondations au rocher, les remblais étant limités aux zones extrêmes, là où le rocher se présentait à faibles profondeurs.

Si ces nouvelles dispositions ont eu pour conséquences fâcheuses d'augmenter très sensiblement le coût de l'ouvrage, elles auront permis de présenter un ouvrage qui, s'il n'améliore pas l'environnement, aura le mérite de beaucoup mieux s'intégrer au site et de limiter l'agression causée par sa construction. 

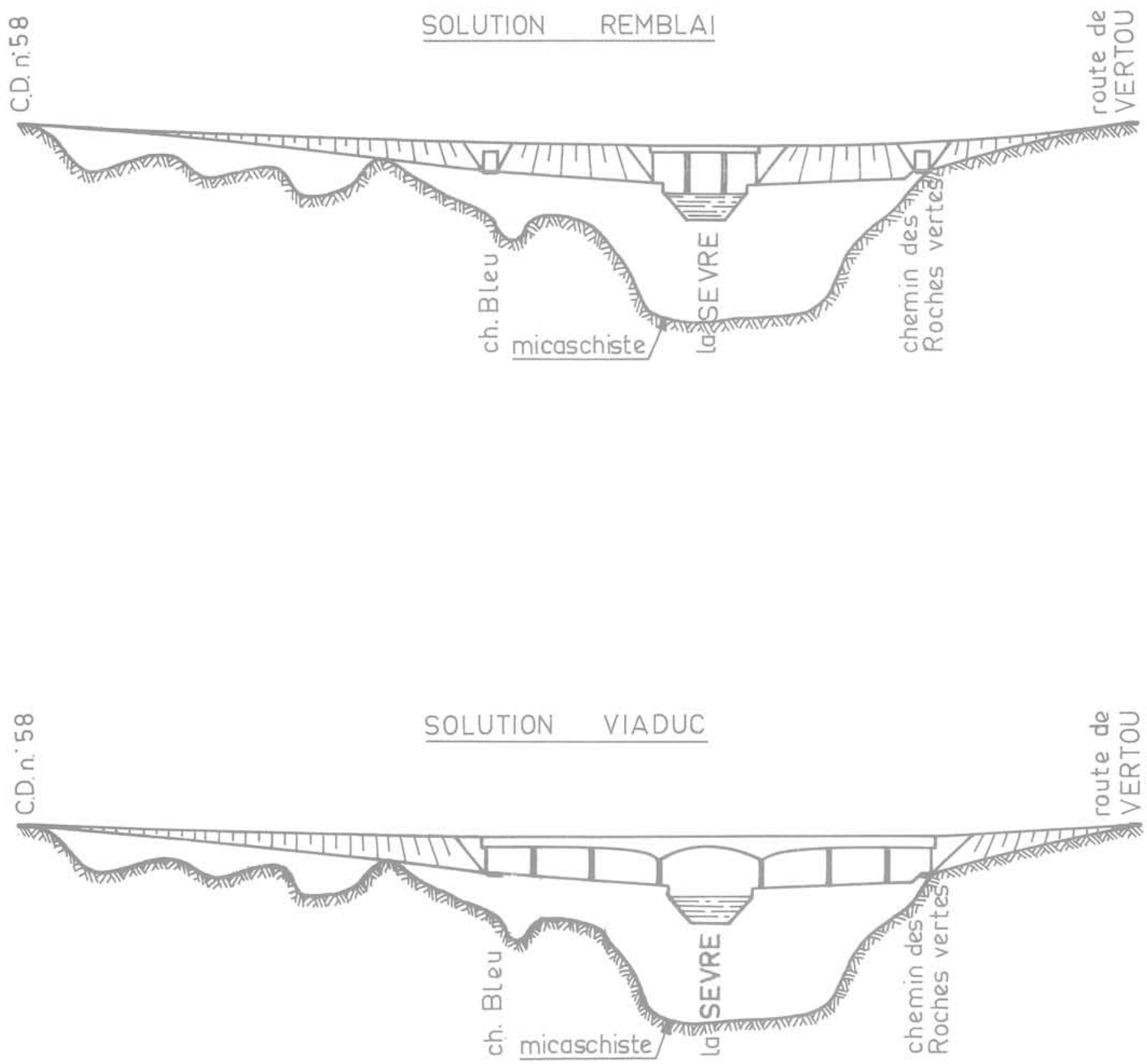
- Le second ouvrage correspond au franchissement de la Loire à Cheviré

Confrontés au triple problème du débit à assurer, au droit du franchissement, à une circulation de voirie rapide, du maintien d'un haut gabarit pour la navigation et de l'incorporation au site de l'ouvrage, nous avons étudié toutes les solutions de pont tournant, basculant, levant, fixe ; en pesant les avantages et les inconvénients de chacune de ces solutions, la réflexion de nos élus s'est portée vers un franchissement sous-fluvial. Les résultats des sondages en cours nous diront si la géotechnique viendra ou non au secours du respect de l'environnement.

J'en arrive - enfin, direz-vous - à ma conclusion. Jusqu'à maintenant, les aménageurs que nous sommes, faute de crédits d'études - mais n'étaitce pas là un mal français - sommes allés un peu à l'aventure avec, parfois, pour obligation conséquente d'improviser, au lieu de prévoir. Héritiers du passé ou de notre propre manque d'information, nous avons eu à faire face à des problèmes qui auraient pu être ressentis beaucoup plus tôt. Nous étions conscients de l'influence fondamentale de la nature des sols sur la solution de nos problèmes, mais la tendance était, en France, de laisser aux entreprises le soin et la charge financière des études complémentaires, préalables à l'établissement de leurs projets. Ce procédé a coûté fort cher aux Français, et ce, depuis des générations.
Depuis quelques années - et j'en remercie notre Municipalité - il a été admis que des études préalables poussées - comme l'avaient compris, bien avant nous, les Américains - si elles étaient d'un prix de revient apparemment élevé, permettaient d'abaisser le coût des réalisations. Il est évident que le géologue et le géotechnicien doivent intervenir, très en amont, avant l'étude de tout projet d'aménagement, pour nous permettre de tenir compte des contraintes naturelles.

Parfaitement conscient de cette nécessité, je m'associerai pleinement aux propos que M. Bourrelier, Directeur Général du B.R.G.M., a tenu, le 14 mars 1979, à Lyon. "Dans l'esprit de nombreux responsables, l'importance des études préalables est sous-estimée. Il est important de montrer que des erreurs graves peuvent être évitées en tenant compte, au bon moment, c'est-à-dire avant le lancement des projets, des multiples contraintes imposées par le milieu naturel.

Dans un domaine aussi complexe, la création de " banques de données " sera, souvent, nécessaire, mais ne sera jamais suffisante. Rien ne pourra remplacer le travail d'équipes pluridisciplinaires, formées en tenant compte de l'importance et de la difficulté des problèmes à résoudre " et, j'ajouterai, au sein desquelles le géotechnicien doit obligatoirement trouver place. 